A clear asset of the study was the application of multivariate statistics - Principal Component Analysis - to assess, in an integrated manner, the interrelationships between variables related to stress, emotion, and cognition, with the aim of underscoring the sets of variables that form coherent subsets. The advantage of the approach over simpler correlational analyses is clear when exploring the nature of interactions in functional systems, which as stress, emotion, and cognition, might not only interact but also be the phenomenological expression of common brain networks. Strikingly, in the study Principal Component Analysis indicated that higher stress-sensitivity contributes positively to cognitive performance in the selected spatial learning task - a holeboard, that can be surprising if contrasted with "static" models of stressemotion-cognition interactions, which would predict high stress-responsiveness to be associated to impaired performance in associative tasks. However, this is precisely one of the crucial contributions of the study by Brinks et al. (2007), to show that high stressreactive individuals $(\mathrm{BALB} / \mathrm{c})$ can have an advantage over low stress-reactive individuals $(\mathrm{C} 57 \mathrm{BL} / 6 \mathrm{~J})$ to learn about certain relational tasks. As BALB/c mice in the study were superior learners in the modified holeboard while they were previously shown to underperform C57BL/6J mice in the more stressful spatial learning water maze task, a linear relationship cannot explain the association between stress reactivity traits and perform- ance in relational tasks. Instead, Brinks et al. (2007) work - together with earlier reports supports the existence of an inverted $U$-shape to account for spatial learning under stress (Sandi and Pinelo-Nava, 2007). Presumably, the stress added by water immersion in the water maze task would render the "more stressed" BALB/c mice less cognitively effective to solve the stressful spatial challenge. Under such conditions, low stress-responsive individuals (such as C57BL/6J mice) would be benefited by the stress added by the physical characteristics of the task (Sandi et al., 1997). The emerging view underscores the individual stress response (and glucocorticoids as a plausible index; de Kloet et al., 1999; Roozendaal et al., 2002) as a critical factor (as opposed to the stressful context) to relate to learning performance.

The importance of taking individual differences into account to understand the neurobiological systems that mediate particular behaviors is becoming increasingly evident, even if such an approach is still far from being systematically incorporated. Brinks et al. (2007) study provides a clear example of its relevance and shows the benefit of using multivariate analysis approaches to understand complexity related to the neurobiology of behavior. The immediate challenge would be to formulate working models that permit organizing and manipulating such rich complexity in an effective and instructive manner for understanding how stress, emotion, and cognition intimately interact.

\section{REFERENCES}

Brinks, V., van der Mark, M., de Kloet, R., and Oitzl, M. (2007). Emotion and cognition in high and low stress sensitive mouse strains: a combined neuroendocrine and behavioral study in BALB/c and C57BL/6J mice. Front. Behav. Neurosci. 1, 8. doi: 10.3389/ neuro.08/008.2007.

de Kloet, E. R., Oitzl, M. S., and Joëls, M. (1999). Stress and cognition: are corticosteroids good or bad guys? Trends Neurosci. 22, 422-426.

Pessoa, L. (2008). On the relationship between emotion and cognition. Nat. Rev. Neurosci. 9, 148-158.

Phelps, E. A., and LeDoux, J. E. (2005). Contributions of the amygdala to emotion processing: from animal models to human behavior. Neuron 48, 175-187.

Richter-Levin, G., and Akirav, I. (2003). Emotional tagging of memory formation - in the search for neural mechanisms. Brain Res. Rev. 43, 247-256.

Roozendaal, B., Quiriarte, G. L., and McGaugh, J. L. (2002). Glucocorticoids interact with the basolateral amygdala beta-adrenoceptor - cAMP/cAMP/PKA system in influencing memory consolidation. Eur. J. Neurosci. 15, 553-560.

Sandi, C., Loscertales, M., and Guaza, C. (1997). Experience-dependent facilitating effect of corticosterone on spatial memory formation in the water maze. Eur. J. Neurosci. 9, 637-642.

Sandi, C., and Pinelo-Nava, M. T. (2007). Stress and memory: behavioral effects and neurobiological mechanisms. Neural. Plast. PMID: 18060012.

Received: 08 October 2008; published: 15 December 2008

Citation: Front. Neurosci. (2008) 2, 2: 134-135. doi: 10.3389/neuro.01.042.2008

Copyright: (C) 2008 Sandi. This is an open-access publication subject to an exclusive license agreement between the authors and the Frontiers Research Foundation, which permits unrestricted use, distribution, and reproduction in any medium, provided the original authors and source are credited.

\title{
Perinatal choline supplementation: a smart lifelong solution to age-related dementia
}

\author{
Ranier Gutierrez ${ }^{1 *}$ and Sidney A. Simon ${ }^{2}$ \\ Department of Pharmacology, CINVESTAV del IPN, Mexico City, Mexico \\ 2 Department of Neurobiology, Duke University, NC, USA \\ *Correspondence: ranier@cinvestav.mx
}

\section{A commentary on}

Developmental periods of choline sensitivity provide an ontogenetic mechanism for regulating memory capacity and age-related dementia

by Warren H.Meck, Christina L. Williams, Jennifer M. Cermak and Jan Krzysztof Blusztajn
Choline is an essential nutrient that is found in several foods (e.g. eggs and liver), and is synthesized in the body. Choline is an important constituent of phosphatidylcholine, a type of phospholipid found in membranes. As such, choline plays an important role in membrane structure and in membrane-mediated cell signaling via phospholipases. As a precursor to acetylcholine, and a neurotransmitter involved in muscle control, attention, learning and memory, it is important in many cognitive processes. Choline also plays a number of roles in brain development. Maternal reserves of choline 
are depleted during pregnancy and lactation, and precisely at this time the availability of choline for optimal brain development is critical (Zeisel, 2004).

Recently, Meck et al. (2008) showed that supplementation with dietary choline during rat embryonic days ED12-17 and postnatal days PD16-30 prevents the spatial memory decline that is normally observed in aged rats (see Figure 1). To measure spatial memory, the authors used an elevated 12-radial-arm maze (a hippocampaldependent task), where eight arms contain a food pellet and other four arms are always empty. The task of the animal is to retrieve all food pellets using the fewest number of choices. Two types of errors can occur in the task. Re-entries into a baited (foodcontaining) arm are considered to be working memory errors, since the status changes from baited to unbaited, whereas entry to a never-baited arm is considered to be a reference memory error. Remarkably, Meck et al. (2008) found that choline supplementation in the two critical periods reduced the frequency of both types of errors. However, the supplementation with choline during the ED12-17 period caused a greater improvement on spatial memory than the supplementation during the PD16-30 period, indicating that the choline availability during pregnancy is more important for memory enhancement than lactation.

Meck et al. (2008) also studied whether perinatal choline supplementation is also important for optimal brain development in behaviorally naïve rats. In older rats, this was accomplished by counting the number of dendritic spines in the hippocampus, a region involved in the acquisition of spatial memories that also underwent synaptic plasticity (Ramirez-Amaya et al., 1999; Shapiro and Eichenbaum, 1999). Compared with untreated rats, the older rats that received choline during ED12-17 and PD16-30 showed a greater number of dendritic spines in the CA1 and dentate gyrus of the hippocampus. Additional morphologic analysis of granule cells of the dentate gyrus revealed that their dendritic tree shapes were more spread in perinatal choline-treated older rats than the untreated control group. However, as the spread of the dendritic trees of granule cells tends to decrease with age, the result indicates that the perinatal choline supplementation also prevents age-related morphological changes.
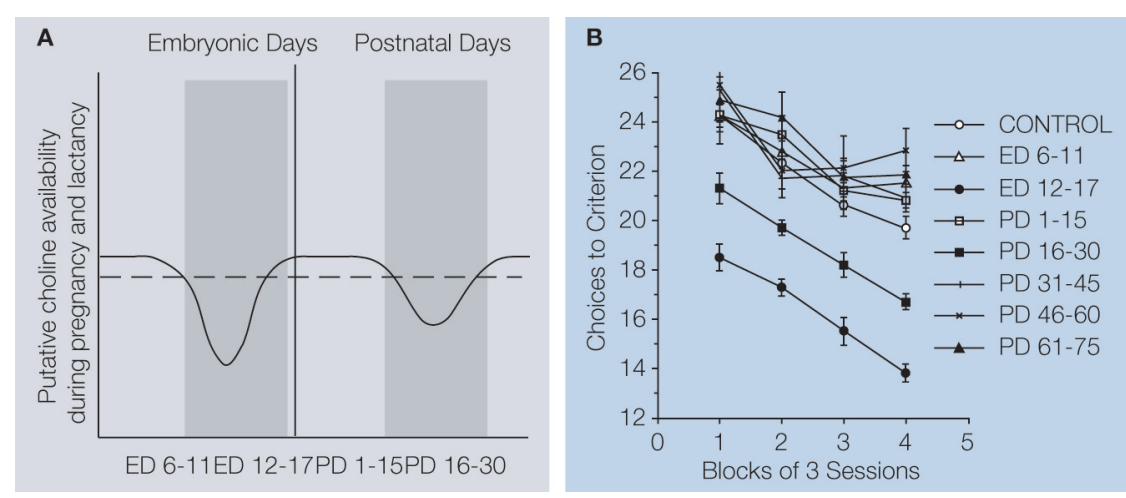

Figure 1 | (A) Schematic representation of two critical periods in which choline deficiency is naturally induced by choline demands imposed by brain development during pregnancy and lactation (Dashed line represents the choline required for optimal rat brain development). The work of Meck et al. (2008) showed that choline supplementation either during Embryonic Days, ED 12-17 or Postnatal Days, PD 16-30 inoculates against the memory decline normally observed in aged rats. (B) Behavioral performance of old rats trained in a spatial memory task as a function of choline supplementation during different developmental periods. Rats that had choline supplemented in ED 12-17 or PD 16-30 solved the 12-radial-arm maze in fewer choices than the counterparts that did not receive choline (control) or received it in other time durations.

Finally, Meck et al. (2008) conducted a longitudinal study, to examine whether memory facilitation by prenatal choline supplementation lasts into old age, in which rats that received choline supplementation in the ED12-17 period and control rats (untreated) were trained at 2, 14, and 26 months. Despite the fact that control rats received the same amount of overtraining in the task, choline-supplemented animals exhibited a significantly greater behavioral performance. The result indicates that the choline supplementation has a lifelong effect on brain function. It also suggests that overtraining, at least in the amount used in the experiment, does not rescue the normal memory decline observed with aging. As choline is a precursor for acetylcholine (ACh), Meck et al. (2008) also measured the ACh content in hippocampal slices from animals trained at three different durations. The authors found that the ACh content correlates with the behavioral performance; that is the older rats that made fewer errors had a greater hippocampal ACh content. Therefore, the choline supplementation in the ED12-17 period caused a lifelong increase in cholinergic metabolism, at least, in the hippocampus, and these changes correlate with the behavioral performance. Although there is no doubt about the important role that choline plays in enhancing memory function, it is well known that there are several classes of memories (i.e., non-hippocampal-dependent memories) suggesting that additional studies should be conducted to determine the extent to which choline supplementation protects from normal age-related decline of non-spatial memories. Nevertheless, the study strongly indicates that spatial memory in aged rats can be, in part, determined by what one's mother eats during critical times in pregnancy. Clearly, we hope that before our birth our mothers ate plenty of eggs and liver.

\section{REFERENCES}

Meck, W. H., Williams, C. L., Cermak, J. M., and Blusztajn, J. K. (2008) Developmental periods of choline sensitivity provide an ontogenetic mechanism for regulating memory capacity and age-related dementia. Front. Neurosci. 1, 1-11.

Ramirez-Amaya, V., Escobar, M. L., Chao, V., and Bermudez-Rattoni, F. (1999). Synaptogenesis of mossy fibers induced by spatial water maze overtraining. Hippocampus 9, 631-636.

Shapiro, M.L., and Eichenbaum,H. (1999).Hippocampus as a memory map: synaptic plasticity and memory encoding by hippocampal neurons. Hippocampus 9 , 365-384.

Zeisel, S. H. (2004). Nutritional importance of choline for brain development. J. Am. Coll. Nutr. 23, 621S-626S.

Received: 07 October 2008; published: 15 December 2008 Citation: Front. Neurosci. (2008) 2, 2: 135-136. doi: 10.3389/neuro.01.040.2008

Copyright: (C) 2008 Gutierrez and Simon. This is an openaccess publication subject to an exclusive license agreement between the authors and the Frontiers Research Foundation, which permits unrestricted use, distribution, and reproduction in any medium, provided the original authors and source are credited. 